\title{
Análise do Método Multi-Passos com Transformada Diferencial Generalizada na Modelagem Fracionária
}

\author{
L. K. B. KURODA ${ }^{1}$, A. BRUNO-ALFONSO ${ }^{2}$, P. F. A. MANCERA ${ }^{3}$ e R. F. CAMARGO ${ }^{2}$ \\ Recebido em 19 de dezembro de 2017 / Aceito em 11 de novembro de 2018
}

\begin{abstract}
RESUMO. Apresenta-se uma análise crítica de uma técnica numérica que tem sido usada na resolução de equações diferenciais de ordem fracionária com derivadas de Caputo. Trata-se do método multi-passos com transformada diferencial generalizada. Verifica-se que a versão do método disponível na literatura produz soluções erradas a partir do segundo passo, isto é mostrado em aplicações aos modelos de Malthus e de Riccati. O problema é explicado em termos da não localidade da derivada de Caputo e das propriedades da transformada diferencial generalizada.
\end{abstract}

Palavras-chave: cálculo fracionário, modelagem fracionária, método da transformada diferencial generalizada.

\section{INTRODUÇÃO}

A Modelagem Matemática é um processo que consiste em traduzir uma situação ou tema do meio em que vivemos para uma linguagem matemática. Essa linguagem, que denominamos Modelo Matemático, pressupõe um conjunto de símbolos e relações matemáticas que representam o fenômeno em questão. Dentre as diferentes formas de se modelar um problema, destacamos a feita a partir de equações diferenciais $[17,3]$.

A obtenção de uma equação diferencial cuja solução descreve bem a realidade traz grande dificuldade. Quanto mais próximos estamos de descrever um problema real, maior será o número de variáveis envolvidas e a complexidade das equações. Dentre as modelagens alternativas, o Cálculo de Ordem Não Inteira, tradicionalmente conhecido como Cálculo Fracionário (CF), que é o ramo da matemática que estuda integrais e derivadas de ordens não-inteiras, desempenha um papel de destaque $[6,14]$.

\footnotetext{
*Autor correspondente: Lucas K. B. Kuroda - E-mail: luaskuroda hotmail.com - http://orcid.org/ 0000-0002-3089-6229

${ }^{1}$ UNESP - Universidade Estadual Paulista, Programa de Pós-graduação em Biometria, Botucatu, SP. E-mail: lucaskuroda@ibb.unesp.br, rubens@fc.unesp.br

${ }^{2}$ UNESP - Universidade Estadual Paulista, Faculdade de Ciências, Bauru, SP. E-mail: alexys@fc.unesp.br

${ }^{3}$ UNESP - Universidade Estadual Paulista, Instituto de Biociências, Botucatu, SP. E-mail: paulo.mancera@unesp.br
} 
Considerando uma equação diferencial que descreve um fenômeno, uma maneira comum de usar a modelagem fracionária é substituir as derivadas de ordem inteira por derivadas de ordem não inteira, geralmente com ordem menor que ou igual à ordem das derivadas originais, de modo que a solução do problema original possa ser recuperada como um caso particular [6, 14]. Assim, o principal desafio passa ser buscar técnicas de resolução das equações, por exemplo, se tratando de equações lineares de ordem fracionárias, podemos utilizar a metodologia da transformada de Laplace. Deve-se notar que há limitações na utilizacão dessa metodologia, para modelos são não lineares, que são resolvidas via métodos numéricos, em geral. Entre estes está o método multi-passos com transformada diferencial generalizada (MSGDTM -"Multi-step Generalized Differential Transform Method").

O método MSGDTM foi utilizado para investigar um modelo de abandono do tabagismo, incluindo comparações com método de Runge-Kutta clássico no caso de derivadas de ordem inteira [8]. Aplicado também num modelo epidemiológico para vírus de computador de [10], no estudo da co-infecção por HIV e malária [7] e no tratamento de dinâmica tumoral [2]. Em relação com as soluções do sistema Chua, os autores destacaram que o método tem a vantagem de fornecer uma forma analítica aproximada da solução dentro de cada sub-intervalo de tempo [9].

É importante salientar que uma série de aplicações do método têm sido reportadas, antes da sua validade ter sido rigorosamente verificada. Neste sentido, Momenzadeh et al. [16] apontaram problemas no MSGDTM. A questão é que existe grande risco ao extrapolar, à modelagem de ordem não inteira, os métodos numéricos demonstrados apenas para equações diferenciais de ordem inteira. De um lado, o método da transformada diferencial pode ser generalizado para derivadas de ordem fracionária, do outro lado, o método multi-passos, válido para ordem inteira, mas não se estende de forma automática para o caso fracionário. A não localidade da derivada fracionária e as propriedades da transformada diferencial generalizada devem ser consideradas, uma vez que a derivada fracionária de Caputo é definida em termos de uma integral [6]. Com o intuito de esclarecer a situação, o presente trabalho traz uma análise detalhada do MSGDTM. A análise utiliza uma série de resultados numéricos para os modelos de Malthus e de Riccati [16].

O artigo está organizado da seguinte forma: Na Seção 2, o método multi-passos com transformada diferencial generalizada é descrito. $\mathrm{Na}$ Seçao 3, apresenta os modelos fracionários de Malthus e de Riccati. No primeiro modelo foi encontrado a solução via transformada de Laplace e MSGDTM, já no segundo, utilizamos apenas o método computacional devido à equação ser não linear, dificultando a utilização a transformada de Laplace. Por fim, na Seção 4 são resumidos os principais resultados e as perspectivas da pesquisa.

\section{MÉTODO DA TRANSFORMADA DIFERENCIAL: EXTENSÃO E GENERALIZAÇÃO}

O método da transformada diferencial (DTM) permite resolver uma grande variedade de equações diferenciais de ordem inteira [11, 20, 21]. A transformada diferencial converte cada função (derivável infinitas vezes) na sequência dos seus coeficientes de Taylor. O DTM converte 
a equação diferencial num sistema de equações algébricas para os termos da transformada. Então, a solução é dada por uma série de Taylor, cujo raio de convergência pode ser finito ou infinito. A avaliação numérica da série também pode apresentar instabilidade numérica, limitando mais o intervalo de resolução da equação.

Uma maneira de ampliar o intervalo de resolução de equações diferenciais de ordem inteira, é aplicar o DTM e utilizar multi-passos, ou seja, uma sequência de intervalos. Isto define o método multi-passos da transformada diferencial (MSDTM = Multi-Step Differential Transform Method). Em cada passo, a condição inicial é dada pelos valores finais da solução do passo anterior. Além disso, o tamanho de cada intervalo deve ser menor que o raio de convergência da série de Taylor.

Para resolver equações diferenciais fracionárias com derivada de Caputo de ordem $\beta$, o DTM é generalizado, dando lugar ao Generalized Differential Transform Method (GDTM). A transformada diferencial generalizada de cada função é dada pela sequência dos coeficientes de uma série de potências da mesma, em que os expoentes são múltiplos inteiros não negativos de $\beta$. Novamente, o intervalo de resolução pode ser limitado pelo raio de convergência da série ou por instabilidades numéricas.

Para estender o intervalo de resolução do GDTM, tem sido utilizado um método multi-passos com transformada diferencial generalizada (MSGDTM = Multi-Step Generalized Differential Transform Method) [13, 12, 19]. A ideia é a mesma do MSDTM, mas cada passo utiliza o GDTM. Como comentado antes, esta extensão deve ser verificada e analisada rigorosamente.

A seguir, com o objetivo de contribuir para a modelagem fracionária, são descritos detalhadamente apenas o GDTM e o MSGDTM.

\subsection{Método da transformada diferencial generalizada}

Segundo [12, 19, 18], a técnica da transformada diferencial é um dos métodos numéricoanalíticos para equações diferenciais ordinárias e parciais que utiliza a forma de polinômios como aproximações das soluções exatas que são suficientemente diferenciáveis. A transformada diferencial, $F(k)$, da $k$-ésima derivada da função $f(t)$ é definida como,

$$
F(k)=\frac{1}{k !}\left[\frac{d^{k} f(t)}{d t^{k}}\right]_{t=t_{0}},
$$

Já a transformada inversa de $F(t)$ é dada por $[20,12,19,18,15]$ :

$$
f(t)=\sum_{k=0}^{\infty} F(k)\left(t-t_{0}\right)^{k} .
$$

A correspondência entre as equações (2.1) e (2.2) pode ser evidenciada ao aplicar o resultado da (2.1) na equação (2.2). De fato, seguindo este procedimento temos: 


$$
f(t)=\sum_{k=0}^{\infty} \frac{\left(t-t_{0}\right)^{k}}{k !}\left[\frac{d^{k} f(t)}{d t^{k}}\right]_{t=t_{0}} .
$$

Assim, a transformada diferencial fornece os coeficientes da expansão em série de Taylor. No entanto, as correspondentes derivadas são calculadas iterativamente pelas equações transformadas da função original. Para fins de implementação, a função $f$ é expressa por uma série finita. Assim, a equação (2.2) pode ser escrita como [19, 1, 15],

$$
f(t) \approx \sum_{k=0}^{S} F(k)\left(t-t_{0}\right)^{k}
$$

em que $S$ é suficientemente grande. Para resolver problemas não-lineares utilizando o DTM, precisamos primeiramente aplicar a transformação diferencial (2.1) na equação de estudo, resultando em uma relação de recorrência, depois resolvendo esta equação utilizando a transformada diferencial inversa (2.2), obtemos a solução do problema.

O método de transformada diferencial generalizada (GDTM) é utilizado para exibir a solução numérica de equações diferenciais de ordem fracionária. $\mathrm{O}$ método proposto é baseado na fórmula generalizada de Taylor. Definimos a transformada diferencial generalizada da k-derivada da função $f(t)$ como [19],

$$
F(k)=\frac{1}{\Gamma(\beta k+1)}\left[\left(D^{\beta}\right)^{k} f(t)\right]_{t=t_{0}},
$$

sendo $0<\beta \leq 1,\left(D^{\beta}\right)^{k}$ a derivada $D^{\beta}$ k-vezes e $D^{\beta}$ a derivada de Caputo de ordem $\beta$, com início em $t=t_{0}[6,4,5]$,

$$
D^{\beta} f(t)=\frac{1}{\Gamma(n-\beta)} \int_{t_{0}}^{t} f^{(n)}(\tau)(t-\tau)^{n-\beta-1} d \tau,
$$

$\operatorname{com} n$ sendo o menor dos inteiros maiores ou iguais que $\beta$.

Seja $D^{\beta}$ a derivada de Caputo (2.4), $f(t)=\left(t-t_{0}\right)^{\alpha}$ e $u=\frac{\tau-t_{0}}{t-t_{0}}$, temos 


$$
\begin{aligned}
D^{\beta}\left(t-t_{0}\right)^{\alpha} & =\frac{\Gamma(\alpha+1)}{\Gamma(\alpha-n+1) \Gamma(n-\beta)} \int_{t_{0}}^{t}\left(\tau-t_{0}\right)^{\alpha-n}(t-\tau)^{n-\beta-1} d \tau \\
& =\frac{\Gamma(\alpha+1)}{\Gamma(\alpha-n+1) \Gamma(n-\beta)} \int_{0}^{1}\left[u\left(t-t_{0}\right)\right]^{\alpha-n}\left[-u\left(t-t_{0}\right)+\left(t-t_{0}\right)\right]^{n-\beta-1}\left(t-t_{0}\right) d u \\
& =\frac{\Gamma(\alpha+1)}{\Gamma(\alpha-n+1) \Gamma(n-\beta)} \int_{0}^{1} u^{\alpha-n}\left(t-t_{0}\right)^{\alpha-n}\left(t-t_{0}\right)^{n-\beta-1}\left(t-t_{0}\right)(1-u)^{n-\beta-1} d u, \\
& =\frac{\Gamma(\alpha+1)\left(t-t_{0}\right)^{\alpha-\beta}}{\Gamma(\alpha-n+1) \Gamma(n-\beta)} \int_{0}^{1} u^{\alpha-n}(1-u)^{n-\beta-1} d u, \\
& =\frac{\Gamma(\alpha+1)\left(t-t_{0}\right)^{\alpha-\beta}}{\Gamma(\alpha-n+1) \Gamma(n-\beta)} B(\alpha-n+1, n-\beta), \\
& =\frac{\Gamma(\alpha+1)\left(t-t_{0}\right)^{\alpha-\beta}}{\Gamma(\alpha-n+1) \Gamma(n-\beta)} \frac{\Gamma(\alpha-n+1) \Gamma(n-\beta)}{\Gamma(\alpha-n+1+n-\beta)} \\
& =\frac{\Gamma(\alpha+1)}{\Gamma(\alpha-\beta+1)}\left(t-t_{0}\right)^{\alpha-\beta}
\end{aligned}
$$

desde que $\alpha>n-1$.

Temos que a transformada inversa de (2.3) é dada por [19],

$$
f(t)=\sum_{k=0}^{\infty} F(k)\left(t-t_{0}\right)^{\beta k}
$$

Vejamos,

$$
\left(D^{\beta}\right)^{0} f(t)=f(t)=F(0)+F(1)\left(t-t_{0}\right)^{\beta}+F(2)\left(t-t_{0}\right)^{2 \beta}+\ldots+F(k)\left(t-t_{0}\right)^{k \beta}+\ldots
$$

Quando $t=t_{0}$,

$\left(D^{\beta}\right)^{0} f\left(t_{0}\right)=F(0)$.

Como visto em (2.5)

$$
\begin{aligned}
\left(D^{\beta}\right)^{1} f(t)= & \frac{F(1) \Gamma(\beta+1)}{\Gamma(1)}+\frac{F(2) \Gamma(2 \beta+1)}{\Gamma(\beta+1)}\left(t-t_{0}\right)^{\beta}+\ldots+ \\
& +\frac{F(k) \Gamma(k \beta+1)}{\Gamma((k-1) \beta+1)}\left(t-t_{0}\right)^{(k-1) \beta}+\ldots
\end{aligned}
$$

Quando $t=t_{0}$,

$$
\begin{aligned}
\left(D^{\beta}\right)^{1} f\left(t_{0}\right)= & F(1) \Gamma(\beta+1) . \\
\left(D^{\beta}\right)^{2} f(t)= & \frac{F(2) \Gamma(2 \beta+1)}{\Gamma(\beta+1)} \frac{\Gamma(\beta+1)}{1}+\frac{F(3) \Gamma(3 \beta+1)}{\Gamma(2 \beta+1)} \frac{\Gamma(2 \Gamma+1)}{\Gamma+1}\left(t-t_{0}\right)^{\beta}+\ldots+ \\
& +\frac{F(k) \Gamma(k \beta+1)}{\Gamma((k-1) \beta+1)} \frac{\Gamma((k-1) \beta+1)}{\Gamma((k-2) \beta+1)}\left(t-t_{0}\right)^{(k-1) \beta}+\ldots
\end{aligned}
$$

Quando $t=t_{0}$,

$\left(D^{\beta}\right)^{2} f\left(t_{0}\right)=F(2) \Gamma(2 \beta+1)$. 
Assim,

$\left(D^{\beta}\right)^{k} f\left(t_{0}\right)=F(k) \Gamma(k \beta+1)$.

Ou seja, como visto em (2.3)

$$
F(k)=\left[\frac{\left(D^{\beta}\right)^{k} f(t)}{\Gamma(k \beta+1)}\right]_{t=t_{0}} .
$$

Substituindo (2.3) em (2.5) temos,

$$
f(t)=\sum_{k=0}^{\infty} \frac{\left(t-t_{0}\right)^{\beta k}}{\Gamma(\beta k+1)}\left[\left(D^{\beta}\right)^{k} f(t)\right]_{t=t_{0}} .
$$

Na Tabela 1, apresentamos algumas propriedades da transformada diferencial generalizada. As duas primeiras exprimem a linearidade da transformada e a terceira expressa a transformada do produto como convolução. A quarta propriedade é fundamental para o GDTM, uma vez que fornece uma fórmula simples da transformada da derivada de Caputo. A demonstração dos resultados tabelados estão no Apêndice ou podem ser vistos em [19].

Tabela 1: Propriedades da transformada diferencial generalizada. Considera-se que $r$ é uma constante e que as transformadas de $x(t)$ e $y(t)$ são $X(k)$ e $Y(k)$, respectivamente, com ordem $\beta$ e centro em $t_{0}$.

\begin{tabular}{ll}
\hline Função Original & Transformada Diferencial Generalizada \\
\hline$f(t)=r x(t)$ & $F(k)=r X(k)$ \\
$f(t)=x(t) \pm y(t)$ & $F(k)=X(k) \pm Y(k)$ \\
$f(t)=x(t) y(t)$ & $F(k)=\sum_{l=0}^{k} X(l) Y(k-l)$ \\
$f(t)=D_{t_{0}}^{\beta} x(t)$ & $F(k)=\frac{\Gamma[(k+1) \beta+1]}{\Gamma(k \beta+1)} X(k+1)$ \\
\hline
\end{tabular}

Consideremos um problema de valor inicial de equação diferencial de ordem fracionária, $\beta$, em relação à função $x(t)$, da forma

$$
D_{t_{0}}^{\beta} x(t)=f(t, x(t)),
$$

em que $f(t, x)$ depende de $t$ e $x$ suavemente, não necessariamente de forma linear. A equação deve ser resolvida para $t>t_{0}, \operatorname{com} x\left(t_{0}\right)=x_{0}$.

Ao aplicar a transformada à condição inicial temos que $X(0)=x_{0}$, enquanto a aplicação à equação diferencial produz

$$
X(k+1)=\frac{\Gamma(k \beta+1)}{\Gamma[(k+1) \beta+1]} F(k, X),
$$


em que $F(k, X)$ é a transformada da composta $f(t, x(t))$. Aqui o símbolo $X$ representa todos os valores da transformada de $x(t)$. Quando $f(t, x)=r x$, a Tabela 1 fornece $F(k, X)=r X(k)$, levando à relação de recorrência

$$
X(k+1)=\frac{r \Gamma(k \beta+1)}{\Gamma[(k+1) \beta+1]} X(k) .
$$

Um outro exemplo é $f(t, x)=2 x-x^{2}+1$, donde

$$
F(k, X)=2 X(k)-\sum_{l=0}^{k} X(l) X(k-l)
$$

$\mathrm{e}$

$$
X(k+1)=\frac{\Gamma(k \beta+1)}{\Gamma[(k+1) \beta+1]}\left[2 X(k)-\sum_{l=0}^{k} X(l) X(k-l)\right] .
$$

\subsection{Método multi-passos com transformada diferencial generalizada}

Nesta seção é apresentado o MSGDTM, de acordo com Arshad et al. [2]. Para resolver o problema de valor inicial num intervalo $\left[t_{0}, T\right]$, a ideia do método multi-passos é dividir o intervalo em $M$ subintervalos da forma $\left[t_{m-1}, t_{m}\right], m=1,2, \ldots, M$, todos de tamanho $h=\frac{T-t_{0}}{M}$. A solução tem a forma

$$
x(t)=\left\{\begin{array}{cc}
x_{1}(t) & \text { se } t \in\left[t_{0}, t_{1}\right], \\
x_{2}(t) & \text { se } t \in\left[t_{1}, t_{2}\right], \\
\vdots & \\
x_{M}(t) & \text { se } t \in\left[t_{M-1}, t_{M}\right],
\end{array}\right.
$$

sendo $x_{1}(t)$ a solução obtida pelo GDTM no intervalo $\left[t_{0}, t_{1}\right]$. Para $m \geq 2$, aplica-se o GDTM no intervalo $\left[t_{m-1}, t_{m}\right]$, usando a condição inicial $u_{m}\left(t_{m-1}\right)=u_{m-1}\left(t_{m-1}\right)$.

Deve-se notar que Arshad et al. [2] expressam $u_{m}(t)$ como uma série generalizada de potências de $t-t_{m-1}$, e não de $t-t_{0}$. Ao mesmo tempo, a derivada de Caputo não é $D_{t_{m-1}}^{\beta}$, senão $D_{t_{0}}^{\beta}$. Acontece que a derivada de Caputo leva em conta toda a história do sistema, o que está relacionado com a sua característica não local. Isto faz com que a aplicação da equação (2.6) seja questionável para $m \geq 2$. A seguir, veremos o que acontece ao aplicar p método MSGDTM em modelos mais simples.

\section{MODELOS MATEMÁtICOS}

O modelo de Malthus é utilizado na modelagem de crescimento de populacional; Supõe-se que a taxa segundo qual a população cresce em um determinado instante é proporcional ao tamanho da população naquele instante, ou seja [6],

$$
\frac{d x}{d t}(t)=r x(t)
$$

sendo $x(t)$ a população no instante $t$ e $r>0$, a constante de proporcionalidade. 


\subsection{Modelagem Fracionária}

O modelo fracionário de Malthus é uma generalização da equação (3.1). Em lugar da primeira derivada, usa-se a derivada de Caputo de ordem $\beta$ com início em $t=0$, sendo $0<\beta \leq 1$. Portanto, vale

$$
D_{0}^{\beta} x(t)=r^{\beta} x(t)
$$

Neste caso, a aplicação do GDTM leva à relação de recorrência na equação (2.7).

Através da metodologia da transformada de Laplace, temos que a solução analítica deste modelo $[6,12]$, é dada por

$$
x(t)=x(0) E_{\beta}\left((r t)^{\beta}\right) .
$$

sendo $E_{\beta}\left(r t^{\beta}\right)$ a função de Mittag-Leffler [6].

A seguir, são comparadas as soluções analíticas com os resultados computacionais, utilizando o método MSGDTM, sendo $r=1$ e $N(0)=1$.
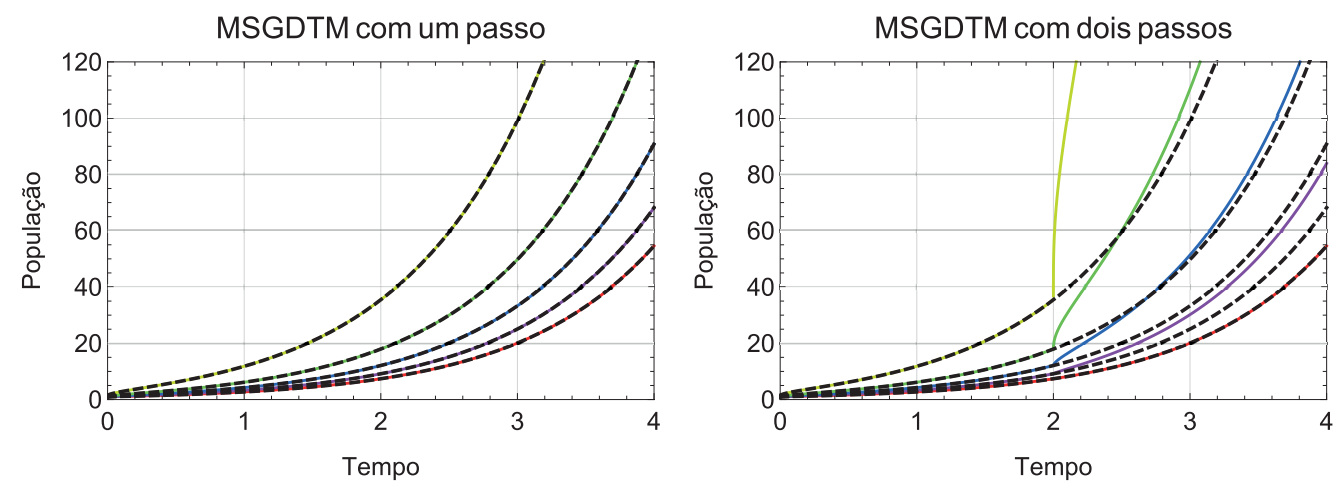

Figura 1: Método MSGDTM e Solução analítica: As linhas tracejadas correspondem à solução analítica, enquanto as linhas contínuas correspondem ao método MSGDTM para $\beta=1, \beta=0.8$, $\beta=0.6, \beta=0.4$ e $\beta=0.2$, respectivamente, de baixo para cima.

Nas Figuras 1 e 2, pode-se notar que o GDTM está aparentemente em bom acordo com a solução analítica da equação (3.2). Ao utilizar o MSGDTM, a solução numérica apresenta singularidades nos pontos da $t_{m}$, com $1 \leq m \leq M-1$. A inexistência de tais singularidades na solução analítica prova que o MSGDTM, como apresentado na literatura, não está correto. De fato, Momenzadeh et al. [16] apontaram a inconsistência da versão atual do método. Com o objetivo de aprofundar mais a análise dos casos considerados por eles, aplicaremos o MSGDTM a um problema de valor inicial da equação de Riccati com derivada de Caputo de ordem $\beta$, sendo $0<\beta \leq 1$, com início em $t_{0}=0$. Com a condição inicial $x(0)=0$, a equação diferencial tem a forma

$$
D_{0}^{\beta} x(t)=2 x(t)-x^{2}(t)+1 .
$$

Nestas condições, o GDTM leva em $X(0)=0$ e na relação de recorrência na equação (2.8). 

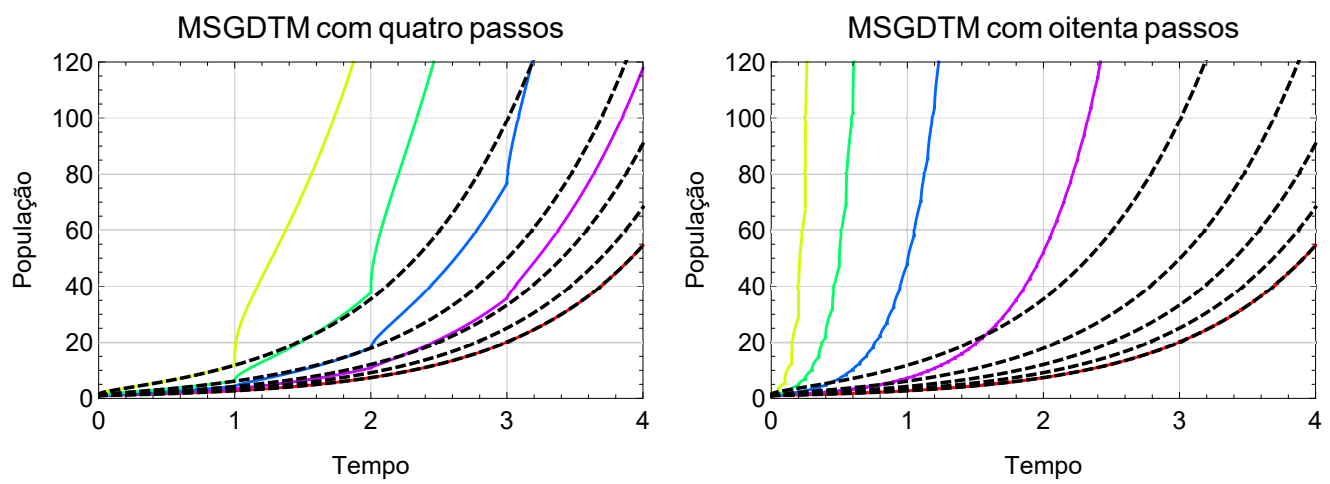

Figura 2: Método MSGDTM e Solução analítica: As linhas tracejadas correspondem à solução analítica, enquanto as linhas contínuas correspondem ao método MSGDTM para $\beta=1, \beta=0.8$, $\beta=0.6, \beta=0.4$ e $\beta=0.2$, respectivamente, de baixo para cima.

Os resultados numéricos para a equação de Ricatti de ordem $\beta=0.7$ foram obtidos no intervalo de $[0,0.4]$. Aplicado o GDTM, temos

$$
x(t) \approx 1.10 t^{0.7}+1.61 t^{1.4}+1.14 t^{2.1}-0.60 t^{2.8}-2.54 t^{3.5}
$$

para $0 \leq t \leq 0.4$. Ao contrário do modelo de Malthus, a equação de Ricatti é não linear, o que dificulta o uso da transformada de Laplace. Assim, neste segundo caso não dispomos de solução alternativa para comparação.

Para o intervalo de tempo entre $0 \leq t \leq 0.4$ existe a convergência do método no modelo de Riccati, no entanto, se estender esse intervalo de solução nota-se que o método deixa de convergir em $t=0.6$ aproximadamente. Por isso, a utilização no método MSDTM é utilizado em modelos com ordem de derivação inteira para resolver o problema de convergência. Neste caso, utilizamos o MSGDTM no modelo de Riccati de ordem $\beta=0.7$.

Dividindo o intervalo em dois, ou seja, o MSGDTM com dois passos de [0,0.2] e [0.2,0.4] teremos como condições iniciais, $y(0)=0$ e $y(0.2) \approx 0.55$. Desta vez, a solução tem a forma

$$
x(t) \approx 1.10 t^{0.7}+1.61 t^{1.4}+1.14 t^{2.1}-0.60 t^{2.8}-2.54 t^{3.5}
$$

para $0 \leq t \leq 0.2 \mathrm{e}$

$$
\begin{aligned}
x(t) \approx & 0.55+1.98(t-0.2)^{0.7}+1.30(t-0.2)^{1.4}-1.54(t-0.2)^{2.1} \\
& -3.07(t-0.2)^{2.8}+0.66(t-0.2)^{3.5}
\end{aligned}
$$

para $0.2 \leq t \leq 0.4$. Na Figura 3 são apresentadas as soluções com um passo e dois passos do método MSGDTM para o problema da equação (3.3). Observa-se que o MSGDTM com dois passos produz uma singularidade em $t=0.2$. Novamente, isto é uma mostra da inconsistência do método. 


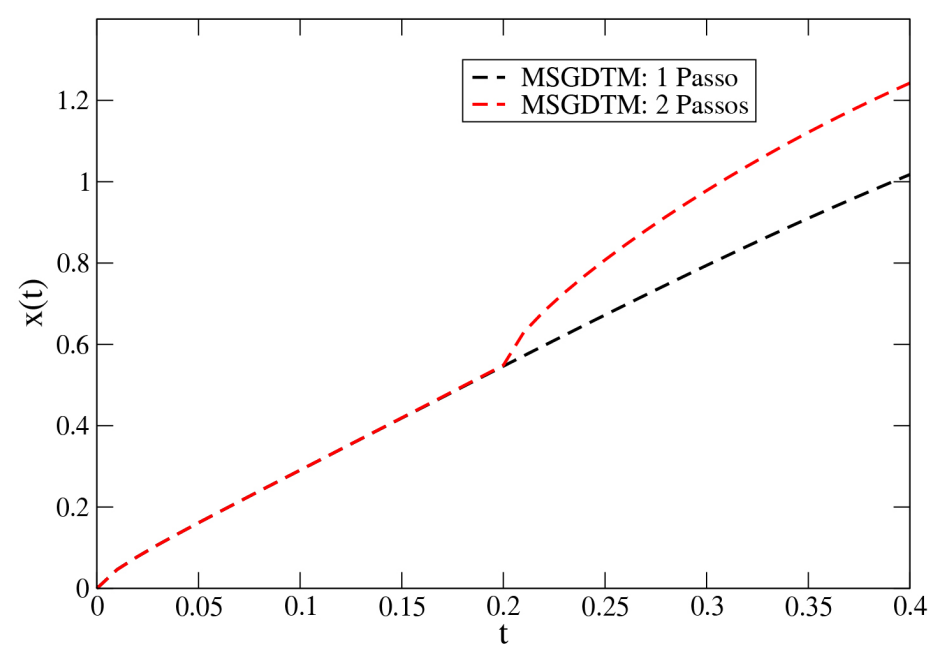

Figura 3: Solução da equação (3.3) utilizando o MSGDTM com um e dois passos.

Resta explicar por que um método inconsistente tem sido amplamente aplicado como se fosse válido. De um lado está a dificuldade de verificar numericamente a qualidade das soluções. Do outro lado está, ao que parece, a evolução do gráfico produzido pelo MSGDTM quando o número de passos, $M$, aumenta suficientemente. Para deixar isto mais claro, divideremos o intervalo em quatro partes, ou seja, aplicaremos o método MSGDTM em 4 passos, [0,0.1], [0.1,0.2], $[0.2,0.3] \mathrm{e}[0.3,0.4]$. As condições iniciais respectivas são $x(0)=0, x(0.1) \approx 0.29, x(0.2) \approx 0.68$ e $x(0.3) \approx 1.12$. As soluções obtidas são

$$
x(t) \approx 1.10 t^{0.7}+1.61 t^{1.4}+1.14 t^{2.1}-0.60 t^{2.8}-2.54 t^{3.5},
$$

para $0 \leq t \leq 0.1$,

$$
\begin{aligned}
x(t) \approx & 0.29+1.65(t-0.1)^{0.7}+1.71(t-0.1)^{1.4}-0.16(t-0.1)^{2.1} \\
& -2.75(t-0.1)^{2.8}-2.53(t-0.1)^{3.5},
\end{aligned}
$$

para $0.1 \leq t \leq 0.2$,

$$
\begin{aligned}
x(t) \approx & 0.68+2.09(t-0.2)^{0.7}+0.97(t-0.2)^{1.4}-2.12(t-0.2)^{2.1} \\
& -2.54(t-0.2)^{2.8}+2.54(t-0.2)^{3.5}
\end{aligned}
$$

para $0.2 \leq t \leq 0.3$

$$
\begin{aligned}
x(t) \approx & 1.12+2.19(t-0.3)^{0.7}-0.37(t-0.3)^{1.4}-2.65(t-0.3)^{2.1} \\
& +1.06(t-0.3)^{2.8}+4.52(t-0.3)^{3.5},
\end{aligned}
$$

para $0.3 \leq t \leq 0.4$. Na Figura 4 representamos graficamente as soluções com um, dois e quatro passos do MSGDTM, aplicado ao problema da equação (3.3). Pode-se observar a presença de 


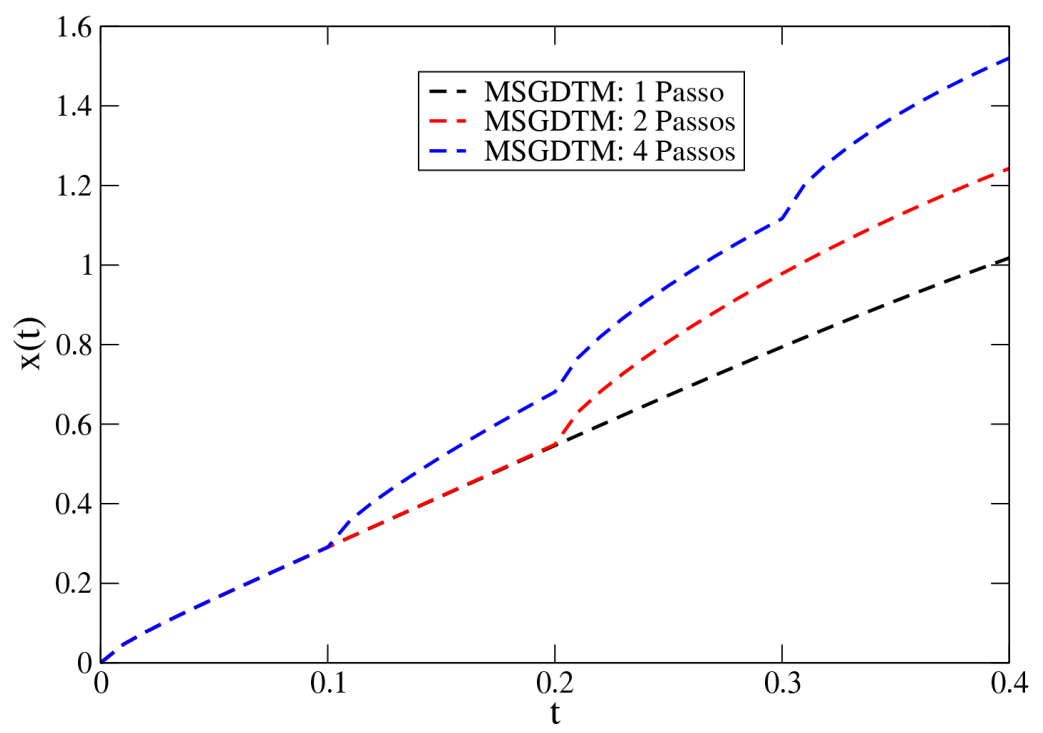

Figura 4: Solução da equação (3.3) utilizando o método MSGDTM com um, dois e quatro passos.

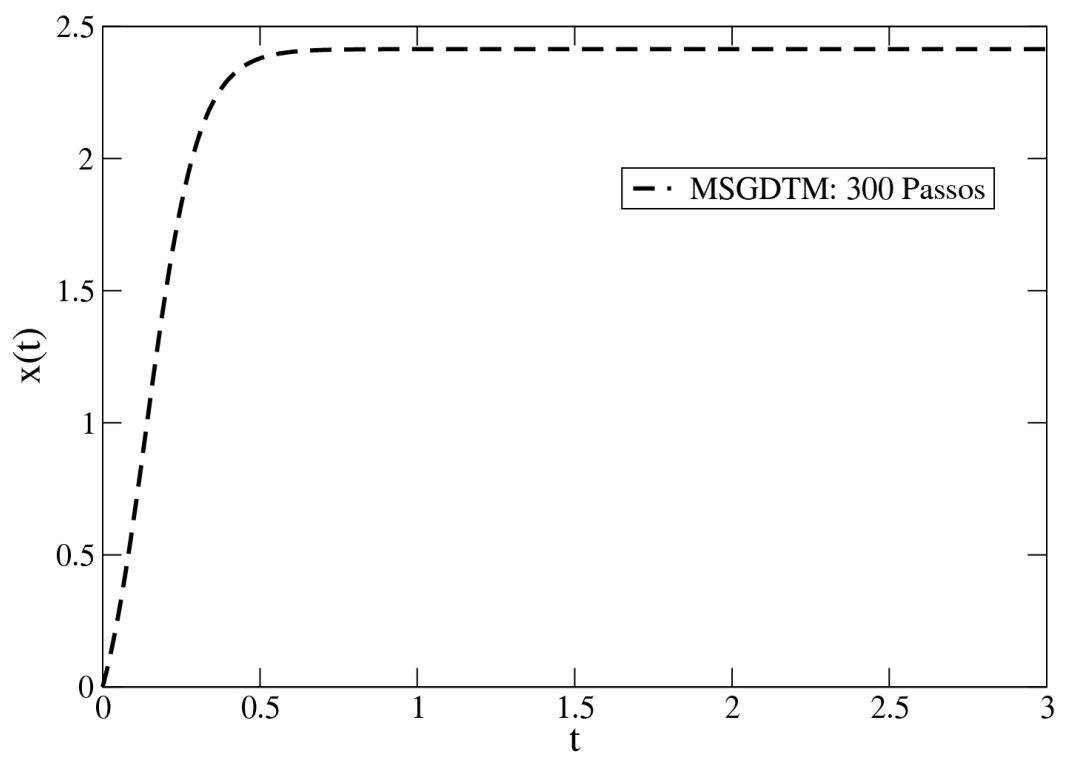

Figura 5: Solução da equação (3.3) utilizando o método MSGDTM com 300 passos no intervalo de $[0,3]$. 
novas singularidades espúrias, ao comparar a curva de quatro passos com a do método GDTM. O resultado para $M=300$, no intervalo $0 \leq t \leq 3$, é mostrado na Figura 5. Desta vez há 299 singularidades espúrias, mas elas não são perceptíveis no gráfico. Aparentemente, o limite da sequência de soluções produzidas pelo MSGDTM converge para uma função suave. Isto pode ter sido interpretado como indício dela ser uma solução do problema.

\title{
4 CONCLUSÕES
}

Foi apresentado e analisado um método multi-passos com transformada diferencial generalizada [2] para a resolução de equações diferenciais de ordem fracionária com derivada de Caputo. Mediante aplicações aos modelos de Malthus e de Riccati, verificou-se que o método é inconsistente na sua versão multi-passos, comparando com a solução obtida pelo método da transformada diferencial generalizada com apenas um passo. A inconsistência é devido à extensão descuidada de técnicas válidas apenas para derivadas de ordem inteira. Tal extensão desconsidera a não localidade da derivada de Caputo e detalhes da propriedade da transformada diferencial generalizada dessa derivada. A aparente convergência das soluções para uma função suave e a carência de soluções exatas para os modelos investigados podem ter dificultado a deteção da inconsistência.

A análise crítica aqui apresentada contribuirá para reforçar o necessário rigor matemático nas diversas aplicações do Cálculo Fracionário. Visando um aporte mais construtivo, correções e alternativas para o método investigado estão em andamento.

\begin{abstract}
This paper presents a critical analysis of a numerical technique that has been used in the resolution of fractional differential equations with Caputo derivative. It is the multi-step method with generalized differential transform. It is found that the version of the method available in the literature produces erroneous solutions from the second step, this is shown in applications to the Malthus and Riccati models. The procedure error is explained in terms of the nonlocality of the Caputo derivative and the properties of the generalized differential transform.
\end{abstract}

Keywords: fractional calculus, fractional modeling, generalized differential transform method, malthusian model.

\section{REFERÊNCIAS}

[1] I.H. Abdel-Halim Hassan. Application to differential transformation method for solving systems of differential equations. Applied Mathematical Modelling, 32 (2008), 2552-2559.

[2] S. Arshad, A. Sohail \& S. Javed. Dynamical study of fractional order tumor model. International Journal of Computational Methods, 12 (2015), 1-12.

[3] R.C. Bassanezi. Modelagem como estratégia metodológica no ensino da matemática. In "Boletim de Educação da SBMAC”. SBMAC, São Paulo (1994).

[4] M. Caputo. Linear Models of Dissipation whose Q is almost Frequency Independent-II. Geophysical Journal International, 13 (1967), 529-539. 
[5] M. Caputo \& M. Fabrizio. A new definition of fractional derivative without singular kernel. Progr. Fract. Differ. Appl, 1(2) (2015), 1-13.

[6] R.F.C. e E. C. Oliveira. "Cálculo fracionário”. Editora Livraria da Física (2015).

[7] B. Ebenezer, K. Awuah-Werekoh \& J. Acquah. Solution of HIV and malaria coinfection model using MSGDTM. Journal of Mathematics Research, 7 (2015), 181-193.

[8] V.S. Erturk, G. Zaman \& S. Momani. A numeric-analytic method for approximating a giving up smoking model containing fractional derivatives. Computers and Mathematics with Applications, 64 (2012), 3065-3074.

[9] A. Freihat \& S. Momani. Application of multistep generalized differential transform method for the solutions of the fractional-order Chua's system. Computers and Mathematics with Applications, 2012 (2012), 1-12.

[10] A.H. Handam \& A.A. Freihat. A new analytic numeric method solution for fractional modified epidemiological model for computer viruses. Applications and Applied Mathematics, 10 (2015), 919-936.

[11] A.S.V.R. Kanth \& K. Aruna. Differential transform method for solving the linear and nonlinear KleinGordon equation. Computer Physics Communications, 180 (2009), 708-711.

[12] L.K.B. Kuroda. "Cálculo fracionário aplicado em dinâmica tumoral: método da transformada diferencial generalizada". Master's thesis, Instituto de Biociências, Universidade Estadual Paulista-UNESP, Botucatu (2016).

[13] L.K.B. Kuroda, A. Bruno-Alfonso, P.F.A. Mancera \& R.F. Camargo. Aplicabilidade do Método da Transformada Diferencial na Modelagem Fracionária. Proceeding Series of the Brazilian Society of Computational and Applied Mathematics, 6(1) (2018), 1-7.

[14] L.K.B. Kuroda, A.V. Gomes, R. Tavoni, P.F.A. Mancera, N. Varalta \& R.F. Camargo. Unexpected behavior of Caputo fractional derivative. Computational and Applied Mathematics, 36(3) (2017), 1173-1183.

[15] F. Mirzaee. Differential transform method for solving linear and nonlinear systems of ordinary differential equations. Applied Mathematical Sciences, 5 (2011), 3465-3472.

[16] A. Momenzadeh \& S. Sarv Ahrabi. A comparison between numerical solutions to fractional differential equations: Adams-type predictor-corrector and multi-step generalized differential transform method. ArXiv e-prints, (2017), 1-12.

[17] D.A. Murray. "Introductory course in differential equations for students in classical and engineering colleges”. Cornell University Library, New York (1902).

[18] Z. Odibat, C. Bertelle, M. Aziz-Alaoui \& G.H.E. Duchamp. A Multi-step differential transform method and application to non-chaotic or Chaotic systems. Applied Mathematical Sciences, 12 (2010), 1-15. 
[19] Z. Odibat, S. Monami \& V.S. Eturk. Generalized differential transform method: Application to differential equations of fractional order. Applied Mathematics and Computation, 197 (2008), 467-477.

[20] Z.M. Odibat. Differential transform method for solving Volterra integral equation with separable kernels. Mathematical and Computer Modelling, 48 (2008), 1144-1149.

[21] B. Soltanalizadeh. Differential transformation method for solving one-space-dimensional telegraph equation. Computational and Applied Mathematics, 30(3) (2011), 639-653. 


\section{APÊNDICE}

Mostraremos a seguir os resultados exibidos da Tabela 1.

1) Pela definição (2.3) e seja $f(t)=r x(t)$,

$$
\begin{aligned}
F(k) & =\frac{1}{\Gamma(\beta k+1)}\left[\left(D^{\beta}\right)^{k} r f(t)\right]_{t=t_{0}}, \\
& =\frac{r}{\Gamma(\beta k+1)}\left[\left(D^{\beta}\right)^{k} f(t)\right]_{t=t_{0}} \\
& =r X(t) .
\end{aligned}
$$

2) Pela definição (2.3) e seja $f(t)=x(t) \pm y(t)$,

$$
\begin{aligned}
F(k) & =\frac{1}{\Gamma(\beta k+1)}\left[\left(D^{\beta}\right)^{k}(x(t) \pm y(t))\right]_{t=t_{0}} \\
& =\frac{1}{\Gamma(\beta k+1)}\left[\left(D^{\beta}\right)^{k} x(t)\right]_{t=t_{0}} \pm \frac{1}{\Gamma(\beta k+1)}\left[\left(D^{\beta}\right)^{k} y(t)\right]_{t=t_{0}}, \\
& =X(t) \pm Y(k) .
\end{aligned}
$$

3) Pela definição (2.5) e seja $f(t)=x(t) y(t)$,

$$
\begin{aligned}
f(t) & =\sum_{k=0}^{\infty} X(k)\left(t-t_{0}\right)^{\beta k} \cdot \sum_{k=0}^{\infty} Y(k)\left(t-t_{0}\right)^{\beta k} \\
& =\left(X(0)+X(1)\left(t-t_{0}\right)^{\beta}+X(2)\left(t-t_{0}\right)^{2 \beta}+\ldots\right) \cdot\left(Y(0)+Y(1)\left(t-t_{0}\right)^{\beta}+Y(2)\left(t-t_{0}\right)^{2 \beta}+\ldots\right) \\
& =X(0) Y(0)+(X(0) Y(1)+X(1) Y(0))\left(t-t_{0}\right)^{\beta}+(X(0) Y(2)+X(1) Y(1)+X(2) Y(0))\left(t-t_{0}\right)^{2 \beta}+\ldots \\
& =\sum_{k=0}^{\infty} \sum_{l=0}^{k} X(l) Y(k-l)\left(t-t_{0}\right)^{\beta k}
\end{aligned}
$$

Logo,

$F(k)=\sum_{l=0}^{k} X(l) Y(k-l)$.

4) Pela definição (2.3) e seja $f(t)=D^{\beta} g(t)$,

$$
\begin{aligned}
F(k) & =\frac{1}{\Gamma(\beta k+1)}\left[\left(D^{\beta}\right)^{k} D^{\beta} g(t)\right]_{t=t_{0}}, \\
& =\frac{1}{\Gamma(\beta k+1)}\left[\left(D^{\beta}\right)^{k+1} g(t)\right]_{t=t_{0}}, \\
& =\frac{\Gamma(\beta(k+1)+1)}{\Gamma(\beta k+1) \Gamma(\beta(k+1)+1)}\left[\left(D^{\beta}\right)^{k+1} g(t)\right]_{t=t_{0}}, \\
& =\frac{\Gamma(\beta(k+1)+1)}{\Gamma(\beta k+1)} G(k+1) .
\end{aligned}
$$

